\title{
Erratum to: Change in decay rates of dioxin-like compounds in Yusho patients
}

Shinya Matsumoto ${ }^{1 *}$, Manabu Akahane ${ }^{1}$, Yoshiyuki Kanagawa' ${ }^{1}$ Jumboku Kajiwara², Chikage Mitoma², Hiroshi Uchi ${ }^{3}$, Masutaka Furue ${ }^{3}$ and Tomoaki Imamura'

\section{Erratum}

n.b. The error described below was the result of a technical error, and thus was not the fault of the authors.

This article [1] contains an error characterised by a discrepancy in the presentation of equation (6) between the article's HTML and PDF formats.

The version of the equation present in the HTML is correct, while conversely the version present in the PDF is incorrect.

As such, the correctly formatted equation is as follows:

$$
Q(t)=\left(\frac{e^{\lambda t} \cdot I}{\lambda}+Q_{0}\right) \cdot e^{-\lambda t}
$$

\footnotetext{
Author details

'Department of Public Health, Health Management and Policy, Nara Medical University School of Medicine, 840 Shijo-cho, Kashihara, Nara 634-8521, Japan. ${ }^{2}$ Fukuoka Institute of Health and Environmental Sciences, 39 Mukaizano, Dazaifu, Fukuoka, Japan. ${ }^{3}$ Department of Dermatology, Graduate School of Medical Sciences, Kyushu University, 3-1-1 Maidashi, Higashi-ku, Fukuoka, Japan.
}

Received: 23 October 2016 Accepted: 27 October 2016

Published online: 16 November 2016

\section{Reference}

1. Matsumoto S, et al. Change in decay rates of dioxin-like compounds in Yusho patients. Environ Health. 2016;15:95. doi:10.1186/s12940-016-0178-0.

\footnotetext{
*Correspondence: shinyamatsumoto-tky@umin.ac.jp

'Department of Public Health, Health Management and Policy, Nara Medical

University School of Medicine, 840 Shijo-cho, Kashihara, Nara 634-8521, Japan
} 